\title{
A genome-skimmed phylogeny of a widespread bryozoan family, Adeonidae
}

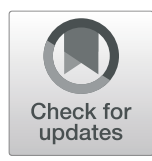

\author{
Russell J. S. Orr ${ }^{*}$ (D, Marianne N. Haugen², Björn Berning ${ }^{3}$, Philip Bock ${ }^{4}$, Robyn L. Cumming ${ }^{5}$, Wayne K. Florence ${ }^{6}$, \\ Masato Hirose ${ }^{7}$, Emanuela Di Martino ${ }^{1}$, Mali H. Ramsfjell', Maja M. Sannum¹, Abigail M. Smith ${ }^{8}$, Leandro M. Vieira9 , \\ Andrea Waeschenbach ${ }^{10}$ and Lee Hsiang Liow ${ }^{1,2^{*}}$
}

\begin{abstract}
Background: Understanding the phylogenetic relationships among species is one of the main goals of systematic biology. Simultaneously, credible phylogenetic hypotheses are often the first requirement for unveiling the evolutionary history of traits and for modelling macroevolutionary processes. However, many non-model taxa have not yet been sequenced to an extent such that statistically well-supported molecular phylogenies can be constructed for these purposes. Here, we use a genome-skimming approach to extract sequence information for 15 mitochondrial and 2 ribosomal operon genes from the cheilostome bryozoan family, Adeonidae, Busk, 1884, whose current systematics is based purely on morphological traits. The members of the Adeonidae are, like all cheilostome bryozoans, benthic, colonial, marine organisms. Adeonids are also geographically widely-distributed, often locally common, and are sometimes important habitat-builders.

Results: We successfully genome-skimmed 35 adeonid colonies representing 6 genera (Adeona, Adeonellopsis, Bracebridgia, Adeonella, Laminopora and Cucullipora). We also contributed 16 new, circularised mitochondrial genomes to the eight previously published for cheilostome bryozoans. Using the aforementioned mitochondrial and ribosomal genes, we inferred the relationships among these 35 samples. Contrary to some previous suggestions, the Adeonidae is a robustly supported monophyletic clade. However, the genera Adeonella and Laminopora are in need of revision: Adeonella is polyphyletic and Laminopora paraphyletically forms a clade with some Adeonella species. Additionally, we assign a sequence clustering identity using cox 1 barcoding region of 99\% at the species and $83 \%$ at the genus level.
\end{abstract}

Conclusions: We provide sequence data, obtained via genome-skimming, that greatly increases the resolution of the phylogenetic relationships within the adeonids. We present a highly-supported topology based on 17 genes and substantially increase availability of circularised cheilostome mitochondrial genomes, and highlight how we can extend our pipeline to other bryozoans.

Keywords: Cheilostome bryozoans, Genome skimming, Mitogenome, Phylogeny, rRNA

\section{Background}

A robust, well-supported species-level phylogeny is often one of the first key steps in understanding evolution above the level of a population. Many understudied taxa have great potential for answering broad evolutionary questions, yet they frequently lack modern phylogenetic treatments. Some of these understudied taxa could also benefit from having their evolutionary relationships

\footnotetext{
*Correspondence: russell_orr@hotmail.com; I.h.liow@nhm.uio.no

${ }^{1}$ Natural History Museum, University of Oslo, Oslo, Norway

Full list of author information is available at the end of the article
}

better resolved in order to inform ecological questions or even management decisions. The phylum Bryozoa is one such taxon. It has been used to study key evolutionary questions [1-3], but as a whole, it is still far behind other comparable taxa in terms of available robust phylogenetic hypotheses estimated using molecular data. As has been shown for other taxa such as damselflies [4], molluscs [5] and fish [6], it is clear that the phylogenetic hypotheses for bryozoans built upon only morphological information are often incongruent with those based on independent molecular data [7-9]. This is

(c) The Author(s). 2019 Open Access This article is distributed under the terms of the Creative Commons Attribution 4.0 International License (http://creativecommons.org/licenses/by/4.0/), which permits unrestricted use, distribution, and reproduction in any medium, provided you give appropriate credit to the original author(s) and the source, provide a link to the Creative Commons license, and indicate if changes were made. The Creative Commons Public Domain Dedication waiver (http://creativecommons.org/publicdomain/zero/1.0/) applies to the data made available in this article, unless otherwise stated. 
because any subset of characters reflects only part of the evolutionary history of the groups in question. There are also strong indications that the integration of different kinds of data gives more information and better estimates of uncertainty of patterns of diversification [10, 11]. Hence, there is a general move towards the integration of morphological, temporal (including fossil) and molecular data in our common quest for understanding phylogenetic relationships including the timing and rates of evolutionary change $[12,13]$. Here, we begin to alleviate the lack of robust phylogenetic hypotheses among bryozoans by focusing our efforts on an ecologically conspicuous family, the Adeonidae.

Adeonidae is a relatively species-rich family whose fossil record is known from Cenozoic seas since the Ypresian, 56.0-47.8 million years ago [14]. Members of this tropical to subtropical family are widespread and often locally abundant. They create habitats that support diverse communities [15], and contribute significantly to shelf carbonate dynamics [16]. This family is currently divided into 10 extant genera with 106 described extant species [17]. Previously, the Adeonidae were separated into two distinct families by Gregory (1893): Adeonidae (including Adeona, Adeonellopsis, Bracebridgia, Cucullipora and Reptadeonella) and Adeonellidae, Gregory 1893 (comprising the genera Adeonella and Laminopora). This separation was corroborated by Cook (1973) who showed that zooids in the Adeonidae exhibit an umbonulomorph development, i.e. zooids in which the polypide and an exposed frontal membrane form during early ontogeny, over which an umbonuloid frontal shield develops at a later stage. In contrast, in the Adeonellidae the zooids show a lepraliomorph development, in which an internal lepralioid frontal shield forms prior to the development of the polypide and ascus. These differences in zooid development and frontal shield formation have historically been considered one of the most important characters in high-level cheilostome systematics (e.g. $[18,19])$, and it was commonly assumed that genera belonging to the same family have the same frontal shield state. However, both types of zooidal development and frontal shields may exist in closely related taxa [19]. For instance, in the genus Parkermavella some species exhibit entirely lepralioid frontal shields while others show mixed types, i.e. partly lepralioid shields with varying extents of umbonuloid areas [20]. These findings dispute the usefulness and stability of this trait for taxonomic classification at a level higher than that of the species (see e.g. [21]). However, testing the monophyly of Adeonidae s.l. using characters independent of morphological traits, i.e. genetic molecular studies, have not been conducted to date. The same applies to the bryozoan order Cheilostomata as a whole: though previous phylogenetic analyses have indicated that the frontal shield may have its limitations as an evolutionary trait that is stable enough to be used in family systematics [7, $22]$, this is yet to be corroborated using a highlysupported tree topology.

Our main aim here is to estimate the phylogenetic relationships among adeonid bryozoans (i.e. Adeonidae s.s. and Adeonellidae s.s.), utilising shot-gun sequencing techniques that have become commonplace among other taxa [23-25]. More recent efforts in building molecular phylogenies of bryozoans, in particular the largest group of extant bryozoans, the cheilostomes, have at most used 7 genes [7, 26-28], using PCR-based methods. Preserved bryozoan material often contains fragmented DNA of the target taxon, making full length amplification problematic. Further, low sequence conservation among bryozoan species impedes the use of "universal primers" for PCR [7, 28].

To alleviate the deficiency of non-morphological data for resolving relationships among species of the Adeonidae s.l., we genome-skimmed (i.e. low coverage genomic shotgun sequencing of a target taxon to recover DNA sequences from high-copy genes) 35 adeonid colonies representing seven named genera (five from Adeonidae s.s. and two from Adeonellidae s.s.) and targeted genes from the mitochondria and (nuclear) ribosomal operon, then built a phylogeny based on these. We discuss to what extent systematics based on adeonid morphology is reflected by their molecular phylogeny and how the latter changes our view of the evolution of this widespread family.

\section{Results}

We successfully sequenced 35 colonies belonging to the Adeonidae, assembled and annotated the majority of the mitochondrial and two rRNA operon genes (Table 1 and Fig. 1). These data are deposited at NCBI with accession numbers. Sixteen of the mitochondrial genomes of the 35 colonies were circularized.

We show the rRNA (Additional file 1: Figure S1) and mitogenome (Additional file 1: Figure S2) ingroup tree inferences to have more congruent topologies than expected by chance with an $I_{\text {cong }}$ index of 2.89 and a $P$ value of 2.82. The result indicates a congruent evolution and supports the concatenation of the rRNA and mitogenome data. As such, we only present the results for the 17 gene datasets in the main text.

Our ingroup taxa form a fully supported monophyletic clade (100 bootstrap (BS) / 1.00 Posterior Probability $(\mathrm{PP})$ ), when the rRNA+mitogenome dataset with outgroup taxa is used for inference (Additional file 1: Figure S3; 43 taxa and 10,259 characters).

The clade consisting of colonies of Adeonella calveti, Adeonella pallasii and A. cf. pallasii from the Mediterranean are the first to diverge within Adeonidae (100BS/ 1.00PP; Fig. 1 based on 35 taxa and 10,531 characters). 
Table 1 Taxa used in this study

\begin{tabular}{|c|c|c|c|c|c|}
\hline Taxon & BLEED nr. & Location & Accession nr. & Circularised mitogenome (bp) & Genes \\
\hline Adeona foliifera fascialis & 298 & W. Aus & MK894382, MK894346, MN131169 & 14,577 & 17 \\
\hline Adeona foliifera fascialis & 297 & W. Aus & MK894378, MK894344, MN131148 & 14,574 & 16 \\
\hline Adeona sp.1 & 746 & W. Aus & MK894379, MN131151 & 14,578 & 15 \\
\hline Adeona sp.1 & 749 & W. Aus & MK894381, MK894345, MN131147 & 14,578 & 16 \\
\hline Adeona sp.2 & 293 & W. Aus & MK894376, MK894342, MN131159 & 14,671 & 17 \\
\hline Adeona sp.2 & 295 & W. Aus & MK894377, MK894343, MN131153 & 14,701 & 17 \\
\hline Adeona sp.3 & 292 & W. Aus & MK894380, MK894375, MN131150 & 14,693 & 17 \\
\hline Adeona sp.4 & 294 & W. Aus & MK894383, MK894374, MN131155 & 14,663 & 17 \\
\hline Adeona sp.5 & 438 & W. Aus & MK894384, MK894347, MN131171 & 16,229 & 17 \\
\hline Adeona sp.5 & 444 & W. Aus & MK894385, MK894348, MN131154 & & 17 \\
\hline Adeona japonica & 49 & $J P$ & MK894386, MK894372, MN131175 & & 17 \\
\hline Adeonellopsis sp.1 & 303 & W. Aus & MK894404, MK894364, MN131166 & & 16 \\
\hline Adeonellopsis sp.2 & 301 & W. Aus & MK894405, MK894365, MN131149 & & 17 \\
\hline Adeonellopsis cf. australis & 300 & W. Aus & MK894402, MK894362, MN131167 & & 17 \\
\hline Adeonellopsis sp.3 & 306 & W. Aus & MK894403, MK894363, MN131172 & & 13 \\
\hline Adeonellopsis sp.4 & 344 & $\mathrm{NZ}$ & MK894406, MK894366, MN131174 & & 17 \\
\hline Adeonellopsis sp.4 & 48 & NZ & MK894407, MK894367, MN131152 & 15,198 & 17 \\
\hline Bracebridgia sp. & 750 & W. Aus & MK894390, MK894370, MN131163 & & 14 \\
\hline Adeonella cf. lichenoides & 305 & W. Aus & MK894399, MK894355, MN131170 & & 17 \\
\hline Adeonella cf. lichenoides & 439 & W. Aus & MK894396, MK894352, MN131162 & & 17 \\
\hline Adeonella cf. lichenoides & 429 & W. Aus & MK894398, MK894353, MN131146 & & 17 \\
\hline Adeonella sp.1 & 416 & W. Aus & MK894397, MK894354, MN131144 & & 17 \\
\hline Adeonella sp.2 & 417 & W. Aus & MK894395, MK894351, MN131176 & & 16 \\
\hline Adeonella pluscula & 393 & SA & MK894391, MK894349, MN131157 & 19,241 & 17 \\
\hline Adeonella pluscula & 410 & SA & MK894392, MK894350, MN131161 & 19,436 & 16 \\
\hline Laminopora jellyae & 391 & SA & MK894393, MK894357, MN131177-81 & & 13 \\
\hline Laminopora jellyae & 408 & SA & MK894394, MK894358, MN131182-87 & & 15 \\
\hline Laminopora contorta & 373 & CV & MK894400, MK894356, MN131158 & & 17 \\
\hline Reptadeonella bipartita & 401 & $\mathrm{BR}$ & MK894388, MK894369, MN131173 & & 15 \\
\hline Reptadeonella brasiliensis & 396 & BR & MK894389, MK894373, MN131164 & 16,957 & 16 \\
\hline Reptadeonella aff. violacea & 41 & $C R$ & MK894387, MK894371, MN131160 & 14,696 & 17 \\
\hline Cucullipora sp. & 302 & W. Aus & MK894401, MK894368, MN131156 & 18,244 & 16 \\
\hline Adeonella calveti & 38 & ALG & MK894408, MK894359, MN131168 & 16,859 & 17 \\
\hline Adeonella cf. pallasii & 39 & $C Y$ & MK894409, MK894360, MN131145 & & 17 \\
\hline Adeonella pallasii & 40 & $C R$ & MK894410, MK894361, MN131165 & & 16 \\
\hline Bugula neritina & & & NC_010197 & & 15 \\
\hline Celleporella hyalina & & & JN680948, NC_018344 & & 15 \\
\hline Flustra foliacea & & & FJ196110, NC_016722 & & 16 \\
\hline Flustrellidra hispida & & & FJ409601, FJ409577, NC_008192 & & 15 \\
\hline Membranipora grandicella & & & NC_018355 & & 14 \\
\hline Pectinatella magnifica & & & FJ409600, FJ409576, NC_038192 & & 16 \\
\hline Tubulipora flabellaris & & & EU650325, DQ333340, NC_015646 & & 15 \\
\hline Watersipora subtorquata & & & JN680947, JN681042, NC_011820 & & 16 \\
\hline
\end{tabular}

In-group taxa generated for this study are shown in white, whilst outgroup taxa are shown in grey. BLEED stands for Bryozoan Lab for Ecology, Evolution and Development and BLEED numbers are numerical tags for the specimens. Accession $\mathrm{nr}$. refer to those at NCBI. Abbreviations for countries (descending order): W. Aus Western Australia, JP Japan, NZ New Zealand, SA South Africa, CV Cape Verde, BR Brazil, CR Croatia, ALG Algeria, and CY Cyprus. The size, in base pairs (bp), of the mitogenome are only shown if it is closed/circularised. Genes, represents the number of genes, of maximum 17, recovered and used in the alignments for each taxon. For an expanded overview of metadata see Additional file 2: Table S1 


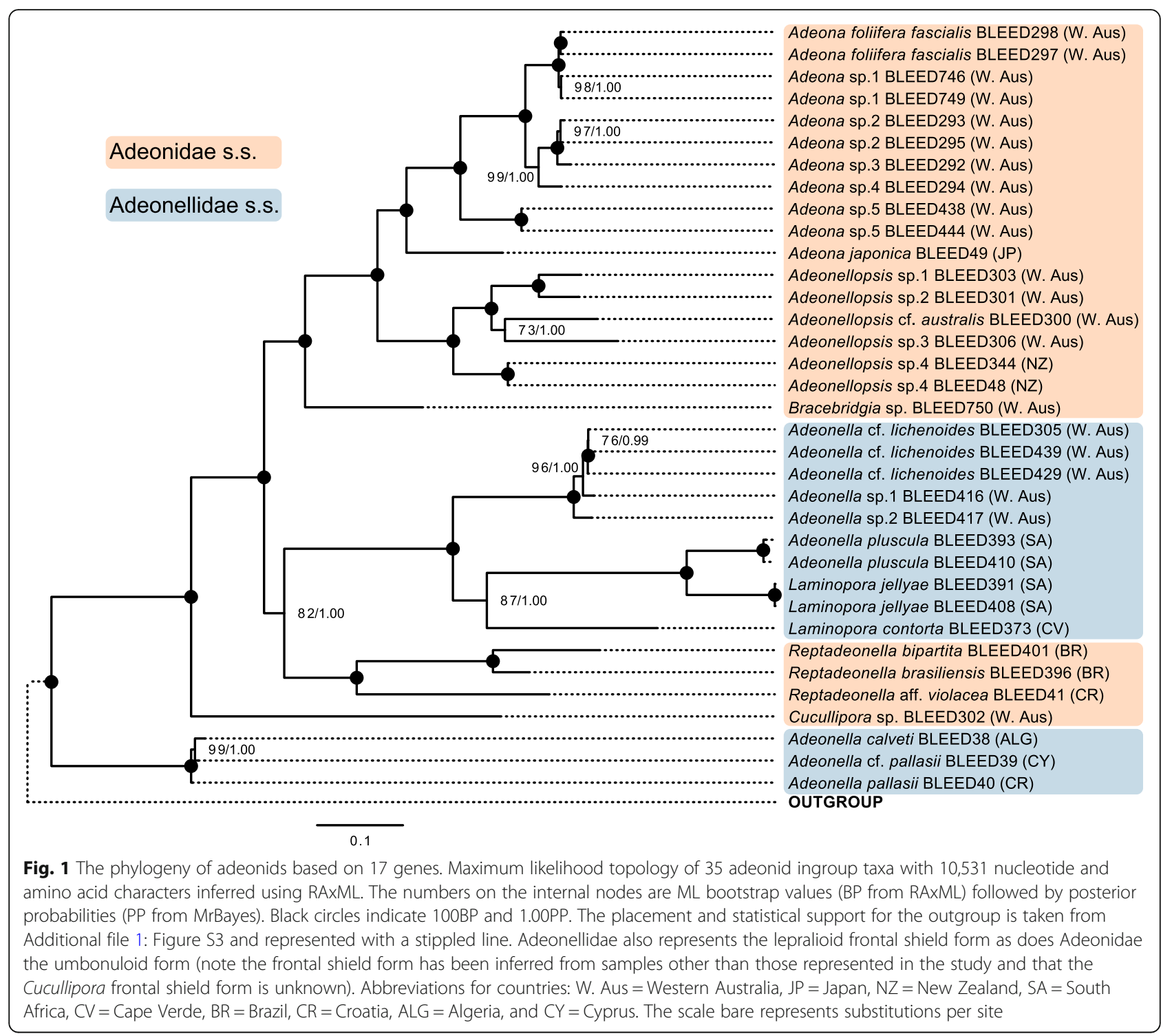

The first taxon to branch off from this basal clade is Cucullipora, excluded from the remaining adeonids with full support (100BS/1.00PP). The latter are divided into two monophyletic clades; The first clade with medium support (82BS/1.00PP) constitutes members of the genera Adeonella, Laminopora, and Reptadeonella. The fully-supported, second clade (100BS/1.00PP) comprises Adeona, Adeonellopsis and Bracebridgia.

In the first clade derived from Cucullipora, three species attributed to the genus Reptadeonella form a fully supported monophyly (100BS/1.00PP), which is sister to the fully-supported clade containing a mixture of Adeonella and Laminopora (100BS/1.00PP). Within the latter, the South African taxa form a fully-supported grouping (100BS/1.00PP) within a larger monophyly (87BS/1.00PP) encompassing Laminopora contorta from Cape Verde. Together with the Western Australian clade of Adeonella
(100BS/1.00PP) this forms a larger monophyletic grouping (100BS/1.00PP).

In the second clade split from Cucullipora, Bracebridgia is inferred as the most basal taxon and excluded from the Adeona and Adeonellopsis monophyly with full support (100BS/1.00PP). The Adeonellopsis clade (100BS/1.00PP), constitutes species from New Zealand and Western Australia. The fully supported Adeona grouping (100BS/1.00PP) includes the Japanese species Adeona japonica (previously Adeonellopsis japonica; see discussion) placing basal to a clade of Western Australian species (100BS/1.00PP).

Clustering at varying levels of nucleotide identity for $18 \mathrm{~s} \mathrm{~V} 4,28 \mathrm{~s}$ D1-D2 and cox1 (Additional file 1: Figure S4) show different levels of sequence conservation. The $18 \mathrm{~s} \mathrm{~V} 4$ region can recover adeonid morphogenera at 99\% clustering identity; 28 s D1-D2 cannot replicate the 
adeonid morpho-taxonomical ranks at any clustering level; but cox1 captures adeonid morphospecies similarities at the 99\% clustering level and genera at $83 \%$ for our dataset.

\section{Discussion}

The interrelationships of adeonids have been controversial throughout the history of bryozoology [29-31], where its constituents have been repeatedly separated and (re)combined in two families, the Adeonidae s.s. and the Adeonellidae. With our new sequence data, we confirm that Adeonidae s.l. is a monophyletic family, regardless of the frontal shield states of its constituent members. The last major comparative morphological study of both adeonid and adeonellid genera was carried out by Cook (1973). Cook concluded that the Adeonellidae (Adeonella and Laminopora) differs radically from the Adeonidae s.s. (Adeona, Adeonellopsis, Reptadeonella and Bracebridgia) in the mode of development of the calcified frontal wall of the autozooid, in the heterochronic development of the polypide and orifice relative to the formation of the frontal shield, and in the homology of the frontal pore complex. However, Cook (1973) also anticipated that, with further studies on adeonids and other cheilostome taxa, the ontogeny of the frontal shield and protrusion apparatus may prove to be phylogenetically uninformative. Comparing the skeletal morphogenesis in the representatives (Adeonella and Adeonellopsis) of the two groups, Lidgard (1996) suggested that their similarities are greater than their differences. We observe that the genera assigned to the two families (the lepraliomorph Adeonellidae and the umbonulomorph Adeonidae s.s.) are distributed across our phylogeny. The Adeonella calveti/pallasii group with its purportedly lepralioid frontal shield forms a basal clade among the taxa we sequenced. The more derived Adeonella clade (which includes the lepraliomorph Laminopora) forms a monophyly with Reptadeonella (umbonulomorph). The clade constituting Bracebridgia, Adeonellopsis and Adeona are also umbonulomorph. With the presumably lepraliomorph group of European Adeonella at the base of the tree, the topology seems to suggest that lepraliomorph development is ancestral, and that umbonulomorph adeonids have evolved at least twice (1: Bracebridgia, Adeonellopsis, Adeona; 2: Reptadeonella; the type of frontal shield development in Cucullipora is unknown at present).

However, the progressive reduction of the umbonuloid component in lepralioid frontal shields over geological time, as well as studies on zooid and frontal shield ontogeny, suggest that lepraliomorph development may be the derived state (e.g. [19, 31]). Given our relatively limited sampling of the adeonids here, it is plausible we have not sampled the most basal extant taxa, which may be umbonulomorph. However, as extant adeonid taxa exhibit derived features (such as endozooidal brooding and extraembryonic nutrition, cf. [32]), and as the genus Adeonella presumably originated already in the Paleogene, it is likely that all potential ancestral lineages are extinct.

We would like to highlight, however, that the above assignments of the umbonulomorph or lepraliomorph states of development to the genera we have sequenced are based on the published literature $[21,31]$ and not the specimens or even the species depicted in our tree. We here abstain from over-interpreting the evolution of frontal shields on our current tree topology for the Adeonidae as further detailed studies on zooid ontogeny and frontal shield formation, particularly of the basal European clade of Adeonella, are necessary for a deeper understanding of the group.

We provide molecular evidence that at least the Adeona species (note that all our samples are from Western Australia) form a statistically supported clade which can be distinguished by their rounded, single-pore spiramen. Its sister clade, Adeonellopsis, which is represented by samples from both Australia and New Zealand, have distinct multiporous spiramina with marginal denticles. Interestingly, what has been called Adeonellopsis japonica has traits intermediate between Adeona and Adeonellopsis, where it appears that only gonozooids have a multiporous spiramen with marginal denticles, but not autozooids. Based on our phylogenetic inference (Fig. 1), Adeonellopsis japonica has a closer genetic affinity to the Adeona group and is better placed in that genus, given the intermediate nature of $A$. japonica's key morphological traits.

Several morphogenera for which we have multiple species represented (Adeona, Reptadeonella, Adeonellopsis) have stood the test of independent molecular data. However, Adeonella did not stand up to the molecular scrutiny. In our statistically highly-supported tree (Fig. 1), Laminopora and the derived Adeonella could be considered a single genus (Additional file 1: Figure S4).

Based on limited available morphological traits and our molecular result, we suggest future studies consider synonymising Laminopora with the derived Adeonella group. Note, however, that the Mediterranean species attributed to Adeonella are at the base of our tree, dissected by Cucullipora, indicating Adeonella as polyphyletic. At this point, we do not have any morphological characters for distinguishing these basal Adeonella from those that are sister to Reptadeonella. The contradiction between morphotype and genotype for the two Adeonella clades demands a taxonomic revision of species attributed to this genus. Until distinguishing traits can be identified with further work, we suggest using molecular markers for assigning extant taxa with 
Adeonella-like morphology to either one of the branches. Further, assigning fossil samples to an Adeonella morphotype should be performed with caution. A substantial amount of work on both the basal group of 'Adeonella' (including A. pallasii, A. cf. pallasii and A. calveti) and the derived group (including $A$. cf. lichenoides and Adeonella sp. 1 and Adeonella sp. 2) is required to detail any distinguishing morphological differences that can be used to delimit these two clades. Given that the basal Adeonella clade contains the type species, the derived clade would either need a new name or formal synonymizing with Laminopora, though the taxonomic work necessary for this is beyond the scope of this current paper. However, the Adeonidae could serve as an example where we might cautiously conclude that morphogenera are, on average, good lineages, although exceptions can occur.

We have used several diverse outgroups to root our adeonid tree (Additional file 1: Figure S3), including a phylactolaemate (Pectinatella), a cyclostome (Tubulipora), a ctenostome (Flustrellidra) and also cheilostomes that are thought to be basal (Membranipora) and more derived with respect to adeonids (Watersipora). However, without wider taxon sampling it is only possible to speculate, based on morphological traits, what the living sister groups to the adeonids might be. In addition to Adeonidae, there is only one other extant family in the superfamily Adeonoidea, the Inversiulidae [33], which would be a natural sister group candidate. Some taxa attributed to Arachnopusiidae also have traits that are reminiscent of Adeonidae, including Poricella, some species of which have adeonid-like spiramina. Some taxa currently placed in the Exechonellidae (e.g. Anarthropora) have occasionally been placed in the Adeonidae [34]. Metrarabdotos (Metrarabdotosidae) and Escharoides (Exochellidae) have likewise been regarded as distant relatives of the Adeonidae [35]. Future studies aiming to further resolve the phylogenetic relationships within the Adeonidae should naturally include the more rarely occurring adeonid genera Triporula, Kubaninella, Anarthropora and Dimorphocella, and should also be more inclusive in the geographic sampling of the genera that we present here.

Clustering three typically used molecular barcoding regions at varying levels of sequence identity allows us to suggest an identity cut-off at the species and genus level, at least for the adeonids we sequenced. Our results suggest that we can use a $99 \%$ clustering identity using the $18 \mathrm{~s}$ V4 region and/or $83 \%$ using cox 1 to delimit adeonid morphogenera; and a $99 \%$ clustering identity using cox 1 to delimit adeonid morphospecies. This differs from the somewhat arbitrary 'universal' clustering level at 97\% identity for species and 90\% for genus (based on $18 \mathrm{~s}$ V4), and reinforces that thresholds need to be determined separately for all groups to avoid either the under- or overestimation of species and genus diversity [36]. Interestingly, if we consider only genetic sequence data, Adeonella pallasii, A. cf. pallasii and A. calveti could be considered as a single species with diverse morphologies (see [37].). Short of carrying out crossing experiments to resolve the biological species problem [38], or common garden experiments such as those done by Jackson and Cheetham for a handful of cheilostomes [2], future studies should consider sequencing more genes from geographically and morphologically diverse representatives of this group of Adeonella. The task of increasing taxon- and gene-sampling naturally applies to other bryozoans as well.

Genome skimming is an effective approach for acquiring enough sequence information for building statistically robust phylogenetic hypotheses that are the bases for further investigations into evolutionary history and processes. Such an approach is relevant for understudied groups whose variation in morphological traits maybe less understood than those of more studied groups, such as mammals and plants. While easily observed morphological traits can give first insights into the systematics of a group, sequence data give many additional and independent characters that can help systematists refine their hypotheses. We show the value of genomeskimmed data for resolving the phylogeny of a cheilostome family for the first time. The ease of extracting whole mitochondrial genomes, for which seven, representing only five species, are currently published in NCBI, [39-44], is also promising.

\section{Conclusion}

Significantly expanding taxon and gene sampling for bryozoans is within reach: we show here that genomeskimming greatly increased the resolution of the phylogenetic relationships within the adeonids, and can be easily extended to other bryozoans. We presented a highly-supported topology based on 17 genes, more than doubling previous best efforts to build bryozoan phylogenies in terms of sequence data [7, 28]. The ease of extracting whole mitochondrial genomes is also promising. As an added bonus, we also note that the taxa sequenced herein are now "barcoded" without using a standard barcoding protocol [45]. Hence, we suggest that Illumina sequencing can be used as an effective alternative to Sanger sequencing, for the barcoding of understudied taxa, such as bryozoans. However, molecular data is not a substitute for detailed taxonomic work and our study should be seen as complementary to taxonomic research. While this study is a small contribution to the genomic data of marine invertebrates, it could be considered a leap in the field of bryozoology. 


\section{Methods and material}

Tissue samples were fixed in 70-95\% ethanol at the time of sampling to minimise DNA degradation. Sequenced material came mainly from Western Australia, with additional samples from Algeria, Brazil, Croatia, Cyprus, Japan, New Zealand and South Africa (Table 1). All samples were exported to Norway according to the protocols of the countries of origin and also adhere to the Nagoya Protocol. Each colony was subsampled for DNA isolation and separately for scanning electron microscopy (SEM), using a Hitachi TM4040PLus. We bleached the latter subsamples in diluted household bleach for a few hours to overnight to remove soft tissues, in order to document skeletal morphology. SEMs of dried samples were taken both pre- and postbleaching (Additional file 3). All physical vouchers are deposited in a public collection at the Natural History Museum of Oslo, University of Oslo, and SEMs are available in the Online Supplementary Information (SI). Other metadata associated with our samples are reported in Additional file 2: Table S1.

\section{DNA isolation, sequencing, assembly and annotation}

Ethanol-preserved samples were dried before genomic DNA was isolated using the DNeasy Blood and Tissue kit (QIAGEN, Germantown, MD, USA). Colonies were homogenised in lysis buffer, using a pestle, in the presence of proteinase-K. Genomic DNA were sequenced at the Norwegian Sequencing Centre (Oslo, Norway) using Illumina HiSeq4000 150 bp paired-end (PE) sequencing with a $350 \mathrm{bp}$ insert size. Illumina HiSeq reads were quality- and adapter-trimmed using TrimGalore v0.4.4 (https://www.bioinformatics.babraham.ac.uk/projects/ trim_galore/) and assembled with SPAdes 3.11.1 [46] with k-mers of $33,55,77,99$, and 121 , before final genome polishing with Pilon [47]. The mitogenome and rRNA operon sequences for all 35 adeonids were identified with blastn in CLC main workbench 7 (Qiagen, Hilden, Germany). Eight bryozoan mitogenomes and 20 nuclear rRNA operons, previously deposited in NCBI were used as queries (Table 1, grey rows).

\section{Annotation and alignments}

The 35 separate mitogenomes were annotated with Mitos2 [48]. There are two rRNA genes (rrnL and rrns) and 13 protein coding genes (atp6, atp8, cox1, cox2, cox3, cob, nad1, nad2, nad3, nad4, nad4l, nad5, and nad6). In addition, two rRNA operon genes (ssu (18 s) and lsu (28 s)) were identified and annotated using RNAmmer [49]. Hence, in total, 17 genes were used for phylogenetic inferences. The eight known bryozoan mitogenomes and rRNA operons (Table 1) were aligned with our 35 adeonids, constituting the out- and in-group taxa respectively. MAFFT [50] with default parameters were used for alignment: for the four rRNA genes (nucleotide), the Q-INS-i model, considering secondary RNA structure, was utilized; for the 13 protein-coding genes, in amino acid format, the G-INS-I model was used. The 17 separate alignments were edited manually using Mesquite v3.1 [51]. Ambiguously aligned characters were removed from each alignment using Gblocks [52] with least stringent parameters.

The single-gene alignments were concatenated using the catfasta2phyml perl script (https://github.com/nylander/catfasta2phyml) into three separate datasets: (i) rRNA operon (two rRNA genes; $18 \mathrm{~s}$ and $28 \mathrm{~s}$ ), (ii) mitogenome (two rRNA genes; rrnS and $\mathrm{rrnL}$ and 13 protein coding genes), and finally (iii) rRNA+mitogenome (four rRNA genes and 13 protein coding genes). Each dataset was inferred with and without the outgroup. The alignments (both masked and unmasked) have been made freely available through the first author's ResearchGate page (https://www.researchgate.net/home) and through Dryad (https://doi.org/10.5061/dryad.7pvmcvdpn).

\section{Phylogenetic reconstruction}

Maximum likelihood (ML) phylogenetic analyses were carried out for each single gene using the "AUTO" parameter in RAxML v8.0.26 [53] to establish the evolutionary model with the best fit. The general time reversible $(G T R+G)$ is the preferred model for the four rRNA genes (18 s, $28 \mathrm{~s}$, rrnS and rrnL), and MtZoa+G for all but one of the 13 protein coding genes, with MtArt $+\mathrm{G}$ being preferred for atp8. The concatenated datasets, divided into gene partitions (above), each with its own separate gamma distribution were analysed using RAxML; The topology with the highest likelihood score of 100 heuristic searches was chosen. Bootstrap values were calculated from 500 pseudo-replicates. Bayesian inference (BI) was performed using a modified version of MrBayes v3.2 [54] incorporating the MtArt and MtZoa evolutionary models (https://github.com/astanabe/mrbayes5d). The datasets were executed, as before, with genes partitions under their separate gamma distribution. Two independent runs, each with three heated and one cold Markov Chain Monte Carlo (MCMC) chain, were initiated from a random starting tree. The MCMC chains were run for 20,000,000 generations with trees sampled every 1000th generation. The posterior probabilities and mean marginal likelihood values of the trees were calculated after the burn-in phase. The average standard deviation of split frequencies between the two runs were $<0.01$, indicating the convergence of the MCMC chains.

Congruence between the topological signal of the bryozoan rRNA operon (Additional file 1: Figure S1) and mitogenome (Additional file 1: Figure S2) ingroup trees was tested, to support their concatenation, using $I_{\text {cong }}$ index [55]. 
As we have samples that belong to the same morphological species, as well as those that are nominally different but closely related, we were interested in exploring criteria for genetic similarity as used in other taxa $[56,57]$ to objectively delimit morphogenera and -species in our dataset. Hence, we ran Cd-hit v4.6.4 [58], a clustering software that groups samples according to sequence identity, with thresholds of $0.99,0.98,0.97,0.95$ and 0.90 on the DNA barcoding regions $18 \mathrm{~s} \mathrm{V4}$ [59], $28 \mathrm{~s}$ D1-D2 [60], and cox1 [56]. Due to its higher interspecies sequence variability, $\operatorname{cox} 1$ was additionally tested with identity thresholds set to 0.80 .

\section{Supplementary information}

Supplementary information accompanies this paper at https://doi.org/10. 1186/s12862-019-1563-4.

Additional file 1: Supporting Figures for Orr et al. Genome skimming the bryozoan tree: lessons from a widespread family, Adeonidae. Figure S1. The phylogeny of adeonids based on two nuclear rRNA genes. Maximum likelihood topology of 35 adeonid ingroup taxa with 5478 nucleotide characters inferred using RAxML. The numbers on the internal nodes are ML bootstrap values (RAxML). The scale bare represents substitutions per site. Figure S2. The phylogeny of adeonids based on 15 mitochondrial genes. Maximum likelihood topology of 35 adeonid ingroup taxa with 5053 nucleotide and amino acid characters inferred using RAxML. The numbers on the internal nodes are ML bootstrap values (RAXML). The scale bare represents substitutions per site. Figure S3. The phylogeny of adeonids based on 17 genes. Maximum likelihood topology of 35 adeonid ingroup taxa and eight outgroup taxa with 10,259 nucleotide and amino acid characters inferred using RAxML. The numbers on the internal nodes are ML bootstrap values (RAxML). Note that data from the outgroup taxa are from NCBI (see Additional file 2: Table S1). The scale bare represents substitutions per site. Figure S4. Adeonid sequence identity for three barcode regions: The topology is identical to that depicted in Fig. 1. The figure shows the inferred adeonid ingroup phylogeny with clustering at varying levels of sequence identity using Cd-hit. 18 s V4 region (99, 98 and 97\% cluster identity) in blue, 28 s D1-D2 regions (99, 97 and 90\% cluster identity) in yellow, and cox1 in (99 and 83\% cluster identity) green. The coloured bars represent the taxa that clustered together at the varying identities, with dots indicating taxa that remained as solitary clusters. The scale bare represents substitutions per site.

Additional file 2. Expanded overview of metadata associated with the samples.

Additional file 3. Scanning Electron Micrographs (SEMs) of dried samples.

\section{Abbreviations}

atp6: Mitochondrially Encoded ATP Synthase Membrane Subunit 6; atp8: Mitochondrially Encoded ATP Synthase Membrane Subunit 8; BS: Bootstrap; cob: cytochrome b; cox1: Cytochrome c oxidase l; cox2: Cytochrome c oxidase 2; cox3: Cytochrome c oxidase 3;

DNA: Deoxyribonucleic acid; LSU (28 s): large subunit; MCMC: Markov Chain Monte Carlo; nad1: NADH dehydrogenase subunit 1; nad2: $\mathrm{NADH}$ dehydrogenase subunit 2; nad3: NADH dehydrogenase subunit 3; nad4: NADH dehydrogenase subunit 4; nad4l: NADH dehydrogenase subunit $4 \mathrm{~L}$; nad5: NADH dehydrogenase subunit 5; nad6: NADH dehydrogenase subunit 6; NCBI: The National Center for Biotechnology Information; PCR: Polymerase chain reaction; PE: Paired end; PP: Posterior probability; RAxML: Randomized Axelerated Maximum Likelihood; rRNA: Ribosomal ribonucleic acid; rrnL: mitochondrial large ribosomal subunit; rrns: mitochondrial small ribosomal subunit; s.l.: sensu lato; s.S.: sensu stricto; SEM: Scanning electron microscopy; SSU (18 s): Small subunit

\section{Acknowledgements}

We thank S. Hageman and an anonymous reviewer for their comments, B.L.G. Thorbek for help with DNA isolation, the Norwegian Sequencing
Centre for the sequencing work, Thomas Schwaha for sample delivery; Ana Carolina Almeida and Adelia Alliz Miranda, Brazil; Katerina Achilleos, Cyprus; Maja Novosel, Croatia for help in collecting samples; the crew of the RV Polaris II and University of Otago general research funding. Specimens from the Kimberley, Western Australia, were collected by the Western Australian Marine Science Institution (WAMSI) as part of the Kimberley Marine Research Program Project 1.1.1 Benthic Biodiversity, funded by the Western Australian government and WAMSI partners, and supported by the Traditional Owners of the Kimberley, the Wunambal Gaambera and Dambimangari Aboriginal Corporations. We thank the masters and crews of RV Solander and RV Linnaeus for their field support. Specimens from Japan were collected by the RV Yayoi (International Coastal Research Center, The University of Tokyo) as part of the research program Tohoku Ecosystem-Associated Marine Science (TEAMS) subsidized by the Ministry of Education, Culture, Sports, Science and Technology (MEXT), Japan.

\section{Authors' contributions}

Research conception and design: RJSO, BB and LHL; Data collection: All authors (RJSO, MNH, BB, PB, RC, WF, MH, EDM, MHR, MMS, AMS, LMV, AW and LHL); Analysis of data: RJSO and MNH; Interpretation of data: RJSO, MNH, BB, EDM, LHL, LV and MH; Drafting the paper: RJSO, MNH and LHL; Critical revision of the paper: All authors; Obtained funding: LMV, AW and LHL. All authors read and approved the final manuscript.

\section{Funding}

This project has received funding from the European Research Council (ERC) under the European Union's Horizon 2020 research and innovation programme (grant agreement No 724324 to L.H. Liow), the Norwegian Research Council (NFR 235073/F2 to L.H. Liow), the Leverhulme Trust Research project grant (award no. RPG-2016-429 to A. Waeschenbach), the Sao Paulo Research Foundation grant (FAPESP 13/50228-8 to L. M. Vieira), and the National Council for Scientific and Technological Development grant (CNPq 308768/2018-3, to L. M. Vieira). The highlighted funding bodies had no involvement in the design of the study, collection, analysis, interpretation of data, and in the writing of the manuscript.

\section{Availability of data and materials}

All sequences produced in this study have been deposited in the NCBI database under the accessions MK894342-MK894410 and MN131144-MN131187. In addition, the alignments used for phylogenetic inference have been made freely available through the authors ResearchGate pages (https://www.researchgate.net/ home) and Dryad (https://doi.org/10.5061/dryad.7pvmcrdpn).

Ethics approval and consent to participate

Not applicable.

\section{Consent for publication}

Not applicable.

\section{Competing interests}

The authors declare that they have no competing interests.

\section{Author details}

${ }^{1}$ Natural History Museum, University of Oslo, Oslo, Norway. ${ }^{2}$ Centre for Ecological and Evolutionary Synthesis, Department of Biosciences, University of Oslo, Oslo, Norway. ${ }^{3}$ Geoscience Collections, Upper Austrian State Museum, Linz, Austria. ${ }^{4}$ Museum Victoria, Melbourne, Victoria, Australia. ${ }^{5}$ Museum of Tropical Queensland, Townsville, Australia. ${ }^{6}$ Department of Research and Exhibitions, Iziko Museums of South Africa, Cape Town, South Africa. ${ }^{7}$ School of Marine Biosciences, Kitasato University, Kanagawa, Japan. ${ }^{8}$ Department of Marine Science, University of Otago, Dunedin, New Zealand. ${ }^{9}$ Department of Zoology, Universidade Federal de Pernambuco, Recife, Brazil.

${ }^{10}$ Department of Life Sciences, Natural History Museum, London, UK.

Received: 6 June 2019 Accepted: 15 December 2019

Published online: 27 December 2019

\section{References}

1. Cheetham AH. Tempo of evolution in a Neogene bryozoan: rates of morphologic change within and across species boundaries. Paleobiology. 1986;12(2):190-202. 
2. Jackson JBC, Cheetham AH. Evolutionary significance of morphospecies - a test with cheilostome Bryozoa. Science. 1990;248(4955):579-83.

3. McKinney FK, Lidgard S, Sepkoski JJ, Taylor PDT. Decoupled temporal patterns of evolution and ecology in two post-Paleozoic clades. Science. 1998;281:807-9.

4. Dijkstra KDB, Kalkman VJ, Dow RA, Stokvis FR, Van Tol J. Redefining the damselfly families: a comprehensive molecular phylogeny of Zygoptera (Odonata). Syst Entomol. 2014;39(1):68-96.

5. Criscione F, Ponder WF, Köhler F, Takano T, Kano Y. A molecular phylogeny of Rissoidae (Caenogastropoda: Rissooidea) allows testing the diagnostic utility of morphological traits. Zool J Linnean Soc. 2017;179(1):23-40.

6. Betancur-R R, Wiley EO, Arratia G, Acero A, Bailly N, Miya M, Lecointre G, Orti G. Phylogenetic classification of bony fishes. BMC Evol Biol. 2017;17:162.

7. Waeschenbach A, Taylor PD, Littlewood DTJ. A molecular phylogeny of bryozoans. Mol Phylogenet Evol. 2012;62:718-35.

8. Taylor PD, Waeschenbach A. Phylogeny and diversification of bryozoans. Palaeontology. 2015;58:85-599.

9. Taylor PD, Waeschenbach A, Smith AB, Gordon DP. In search of phylogenetic congruence between molecular and morphological data in bryozoans with extreme adult skeletal heteromorphy. Syst Biodivers. 2015; 13(6):525-44.

10. Slater GJ, Harmon LJ, Alfaro ME. Integrating fossils with molecular phylogenies improves inference of trait evolution. Evolution. 2012;66(12): 3931-44.

11. Hunt G, Slater G. Integrating paleontological and phylogenetic approaches to macroevolution. Annu Rev Ecol Evol Syst. 2016;47:189-213.

12. Ronquist F, Klopfstein S, Vilhelmsen L, Schulmeister S, Murray DL, Rasnitsyn AP. A total-evidence approach to dating with fossils, applied to the early radiation of the hymenoptera. Syst Biol. 2012;61(6):973-99.

13. Zhang C, Stadler T, Klopfstein S, Heath TA, Ronquist F. Total-evidence dating under the fossilized birth-death process. Syst Biol. 2015;65(2):228-49.

14. Gordon DP, Taylor PD. Bryozoa of the early Eocene Tumaio limestone, Chatham Island, New Zealand. J Syst Palaeontol. 2015;13(12):983-1070.

15. Cumming RL, Gordon DP, Gowlett-Holmes K. In: Cook PL, Bock PE, Gordon DP, Weaver H, editors. Bryozoans in the marine benthos. Australia: CSIRO Publishing; 2018. p. 145-61.

16. Smith AM. Bryozoans as southern sentinels of ocean acidification: a major role for a minor phylum. Mar Freshw Res. 2009;60(5):475-82.

17. Bock PE, Gordon D. Phylum Bryozoa Ehrenberg, 1831. Zootaxa. 2013;3703(1):67-74.

18. Gordon DP. Bryozoan frontal shields: studies on umbonulomorphs and impacts on classification. Zool Scr. 1993;22(2):203-21.

19. Gordon DP. Towards a phylogeny of cheilostomes - morphological models of frontal wall/shield evolution. Balboa: Smithsonian Tropical Research Institute; 2000. p. 17-37.

20. Di Martino E, Taylor PD, Gordon DP, Liow LH. New bryozoan species from the Pleistocene of the Wanganui Basin, North Island, New Zealand. Eur J Taxonomy. 2017:345:1-15.

21. Lidgard S. In: Gordon D, Smith AB, Grant-Mackie JA, editors. Zooid skeletal morphogenesis of some Australian and New Zealand Adeonellopsis (Cheilostomatida); 1996. p. 167-77.

22. Knight S, Gordon DP, Lavery SD. A multi-locus analysis of phylogenetic relationships within cheilostome bryozoans supports multiple origins of ascophoran frontal shields. Mol Phylogenet Evol. 2011:61(2):351-62.

23. Dodsworth S. Genome skimming for next-generation biodiversity analysis. Trends Plant Sci. 2015:9:525-7.

24. Foote AD, Liu Y, Thomas GWC, Vinař T, Alföldi J, Deng J, Dugan S, van Elk $C E$, Hunter ME, Joshi $V$, et al. Convergent evolution of the genomes of marine mammals. Nat Genet. 2015;47:272.

25. Yu C, Kim H, Kim HJ, Jung Y-H. The complete mitochondrial genome of the oriental sea slug: Chromodoris orientalis (Nudibranchia, Chromodorididae). Mitochondrial DNA Part B. 2018;3(2):1017-8.

26. Vieira LM, Winston JE, Fehlauer-Ale KH. Nine new species of Bugula Oken (Bryozoa: Cheilostomata) in Brazilian shallow waters. PLoS One. 2012;7(7): e40492.

27. Fehlauer-Ale KH, Winston JE, Tilbrook KJ, Nascimento KB, Vieira LM. Identifying monophyletic groups within Bugula sensu lato (Bryozoa, Buguloidea). Zool Scr. 2015;44(3):334-47.

28. Orr RJS, Waeschenbach A, Enevoldsen ELG, Boeve JP, Haugen MN, Voje KL, Porter J, Zágoršek K, Smith AM, Gordon DP, et al. Bryozoan genera Fenestrulina and Microporella no longer confamilial; multi-gene phylogeny supports separation. Zool J Linnean Soc. 2018;186:190-9.
29. Gregory JW. On the British Palaeogene Bryozoa. Transact Zool Soc London. 1893;13:219-79.

30. Harmer SF. The Polyzoa of the Siboga Expedition, Part IV. Cheilostomata, Ascophora. II, vol. 28; 1957.

31. Cook PL. Preliminary notes on the ontogeny of the frontal body wall in the Adeonidae and Adeonellidae (Bryozoa, Cheilostomata). Bull British Museum. 1973;25(6):243-63

32. Ostrovsky AN, Gordon DP, Lidgard S. Independent evolution of matrotrophy in the major classes of Bryozoa: transitions among reproductive patterns and their ecological background. Mar Ecol Prog Ser. 2009;378:113-24

33. Cook P, Bock PE, Gordon DP, Weaver H. Australian Bryozoa volume 2: taxonomy of Australian families. Australia: CSIRO; 2018.

34. Hayward PJ, Ryland JS. Cheilostomatous Bryozoa. Part 2. Hippothooidea Celleporoidea. Shrewsbury: Field Studies Council; 1999.

35. Cheetham AH, Sanner J, Jackson JBC. Metrarabdotos and related genera (Bryozoa : Cheilostomata) in the late Paleogene and Neogene of tropical America. J Paleontol. 2007:81(1):1-96.

36. Edgar RC. Updating the $97 \%$ identity threshold for $16 \mathrm{~S}$ ribosomal RNA OTUs. Bioinformatics. 2018;34(14):2371-5.

37. Rosso A, Novosel M. The genus Adeonella (Bryozoa, Ascophora) in the Mediterranean from Pliocene to recent, with description of two new species. J Nat Hist. 2010;44:1697-727.

38. Paterson ID, Mangan R, Downie DA, Coetzee JA, Hill MP, Burke AM, Downey PO, Henry TJ, Compton SG. Two in one: cryptic species discovered in biological control agent populations using molecular data and crossbreeding experiments. Ecol Evol. 2016;6(17):6139-50.

39. Waeschenbach A, Porter JS, Hughes RN. Molecular variability in the Celleporella hyalina (Bryozoa; Cheilostomata) species complex: evidence for cryptic speciation from complete mitochondrial genomes. Mol Biol Rep. 2012;39(9):8601-14.

40. Shen X, Tian M, Meng X, Liu H, Cheng H, Zhu C, Zhao F. Complete mitochondrial genome of Membranipora grandicella (Bryozoa: Cheilostomatida) determined with next-generation sequencing: the first representative of the suborder Malacostegina. Comp Biochem Physiol Part D Genomics Proteomics. 2012;7(3):248-53.

41. Sun MA, Wu Z, Shen X, Ren J, Liu X, Liu H, Liu B. The complete mitochondrial genome of Watersipora subtorquata (Bryozoa, Gymnolaemata, Ctenostomata) with phylogenetic consideration of Bryozoa. Gene. 2009; 439(1):17-24.

42. Waeschenbach A, Telford MJ, Porter JS, Littlewood DTJ. The complete mitochondrial genome of Flustrellidra hispida and the phylogenetic position of Bryozoa among the Metazoa. Mol Phylogenet Evol. 2006;40(1):195-207.

43. Jang KH, Hwang UW. Complete mitochondrial genome of Bugula neritina (Bryozoa, Gymnolaemata, Cheilostomata): phylogenetic position of Bryozoa and phylogeny of lophophorates within the Lophotrochozoa. BMC Genomics. 2009:10:167.

44. Shen X, Tian M, Chu KH, Wang JF, Chen S, Liu HL, Zhao XH, Zhao FQ. Comparative mitogenomic analyses reveal cryptic diversity of the bryozoan Bugula neritina Linnaeus, 1758, in the Yellow Sea. Mar Freshw Res. 2016; 67(8):1241-52.

45. Li J, Yang Q, Huang Q, Fu R, Zhou L, Tang M, Liu S, Su X, Li Y, Zhou X. Ultradeep sequencing enables high-fidelity recovery of biodiversity for bulk arthropod samples without PCR amplification. GigaScience. 2013;2(1):4.

46. Bankevich A, Nurk S, Antipov D, Gurevich AA, Dvorkin M, Kulikov AS, Lesin VM, Nikolenko SI, Pham S, Prjibelski AD, et al. SPAdes: a new genome assembly algorithm and its applications to single-cell sequencing. J Comput Biol. 2012;19(5):455-77.

47. Walker BJ, Abeel T, Shea T, Priest M, Abouelliel A, Sakthikumar S, Cuomo CA, Zeng Q, Wortman J, Young SK, et al. Pilon: an antegrated tool for comprehensive microbial variant detection and genome assembly improvement. PLoS One. 2014;9(11):e112963.

48. Bernt M, Donath A, Jühling F, Externbrink F, Florentz C, Fritzsch G, Pütz J, Middendorf M, Stadler PF. MITOS: Improved de novo metazoan mitochondrial genome annotation. Mol Phylogenet Evol. 2013;69(2):313-9.

49. Lagesen $\mathrm{K}$, Hallin $\mathrm{P}$, Rødland EA, Staerfeldt H-H, Rognes T, Ussery DW. RNAmmer: consistent and rapid annotation of ribosomal RNA genes. Nucleic Acids Res. 2007:35(9):3100-8.

50. Katoh K, Standley DM. MAFFT multiple equence alignment software version 7: improvements in performance and usability. Mol Biol Evol. 2013;30(4): 772-80. 
51. Maddison WP, Maddison DR: Mesquite: a modular system for evolutionary analysis. Version 3.1; 2017. http://mesquiteproject.org.

52. Talavera G, Castresana J. Improvement of phylogenies after removing divergent and ambiguously aligned blocks from protein sequence alignments. Syst Biol. 2007;56(4):564-77.

53. Stamatakis A. RAxML-VI-HPC: maximum likelihood-based phylogenetic analyses with thousands of taxa and mixed models. Bioinformatics. 2006; 22(21):2688-90.

54. Huelsenbeck JP, Ronquist F. MRBAYES: Bayesian inference of phylogenetic trees. Bioinformatics. 2001;17(8):754-5.

55. de Vienne DM, Giraud T, Martin OC. A congruence index for testing topological similarity between trees. Bioinformatics. 2007;23(23):3119-24.

56. Folmer O, Black M, Hoeh W, Lutz R, Vrijenhoek R. DNA primers for amplification of mitochondrial cytochrome c oxidase subunit I from diverse metazoan invertebrates. Mol Mar Biol Biotechnol. 1994:3:294-9.

57. Xiong W, Zhan A. Testing clustering strategies for metabarcoding-based investigation of community-environment interactions. Mol Ecol Resour. 2018;18:1326-38

58. Li W, Godzik A. Cd-hit: a fast program for clustering and comparing large sets of protein or nucleotide sequences. Bioinformatics. 2006;22(13):1658-9.

59. Stoeck T, Bass D, Nebel M, Christen R, Ones MDM, Breiner HW, Richards TA. Multiple marker parallel tag environmental DNA sequencing reveals a highly complex eukaryotic community in marine anoxic water. Mol Ecol. 2010;19(1):S21-31.

60. Sonnenberg R, Nolte AW, Tautz D. An evaluation of LSU rDNA D1-D2 sequences for their use in species identification. Front Zool. 2007:4:6.

\section{Publisher's Note}

Springer Nature remains neutral with regard to jurisdictional claims in published maps and institutional affiliations.

Ready to submit your research? Choose BMC and benefit from:

- fast, convenient online submission

- thorough peer review by experienced researchers in your field

- rapid publication on acceptance

- support for research data, including large and complex data types

- gold Open Access which fosters wider collaboration and increased citations

- maximum visibility for your research: over $100 \mathrm{M}$ website views per year

At BMC, research is always in progress.

Learn more biomedcentral.com/submissions 